\title{
Psicooncología
}

ISSN: 1696-7240

\section{Análisis cualitativo de temas construidos y emergentes a partir de un experiencia piloto de Psicoterapia Centrada en la Compasión (PCC) en pacientes con cáncer avanzado}

\author{
Cristina Prieto Larroya ${ }^{1 *}$; Jacobo Chamorro López ${ }^{2}$; Jordi Fernández Castro ${ }^{3}$; Francisco Luis \\ Gil Moncayo ${ }^{4}$
}

Recibido: 15 de marzo de 2017 / Aceptado: 15 de abril de 2017

Resumen. Introducción: Desde el Instituto Catalán de Oncología de Duran i Reinals, se desarrolla un modelo de Psicoterapia grupal Centrada en la Compasión" (PCC), enfocada a pacientes con cáncer en fase avanzada. El modelo trabaja constructos terapéuticos como "compasión" o "autocompasión", junto a la atención consciente (mindfulness) para mejorar el bienestar emocional y ayudar al crecimiento personal y espiritual de estos pacientes. Objetivo: El presente estudio piloto tiene como objetivo evaluar la adecuación del nuevo modelo de PCC propuesto. Concretamente evalúa la adecuación de los constructos trabajados por la terapia, la existencia de nuevos temas relevantes para el paciente no contemplados en el modelo, y la consistencia global del modelo a nivel de estructura, tareas y contenido. Método: La metodología fue cualitativa, se grabó y transcribió un ciclo de 8 sesiones de PCC y se hizo un análisis temático del contenido de las sesiones. El análisis fue realizado por dos evaluadores de forma independiente. La muestra final estuvo formada por 9 pacientes. Resultado: Los resultados obtenidos indican que hay una buena adecuación de los constructos trabajados en la terapia y del modelo en general. Sin embargo, será necesario incluir el tema de las "relaciones interpersonales", tema muy relevante para el paciente no contemplado en el modelo. Además, se obtuvo información sobre principales preocupaciones de los pacientes, dificultades a la hora de realizar algunas tareas propuestas y un feedback positivo de la propia psicoterapia y de su participación. Conclusiones: Este estudio piloto aporta información relevante que permite ajustar y mejorar la propuesta del modelo de PCC inicial.

Palabras clave: Psicoterapia; compasión; midfulness; cáncer; psicooncología; malestar emocional; investigación cualitativa.

\section{[en] Qualitative analysis of constructed and emerging themes from a pilot experience of Compassion Centered Psychotherapy (PCC) in patients with advanced cancer}

\footnotetext{
Abstract. Introduction: People from the Catalan Institute of Oncology of Duran i Reinals have developed a model of "Psicoterapia grupal Centrada en la Compasión" (PCC), focused on patients with advanced cancer develops. The model works therapeutic constructs as "compassion" or "self-compassion" with

Facultad de Psicología, Universidad Autónoma de Barcelona, Barcelona, España.

Parc Salut Mar, Barcelona, España. E-mail: jchamorrolopez@parcdesalutmar.cat

Facultad de Psicología, Universidad Autónoma de Barcelona, Barcelona, España. E-mail: jordi.fernandez@uab.es

4 Unidad de Psico-Oncología. Institut Català d'Oncología. Hospital Duran i Reynals, Barcelona, España. E.mail: fgil@iconcologia.net

* Dirección de correspondencia: Cristina Prieto Larroya. Facultad de Psicología, Universidad Autónoma de Barcelona. C/Santa Ana, 16. Tamarite de Litera, Huesca. E-mail: cris.prila91@gmail.com
} 
mindfulness to improve emotional distress and help to the personal and spiritual growth of these patients. Objective: This pilot study aims to assess the adequacy of the new model proposed of PCC. Specifically evaluates the adequacy of the constructs therapy worked and the existence of new issues relevant to the patient not included in the model, and the overall consistency of the model for the structure, tasks and content. Method: The methodology was qualitative, one cycle of 8 sessions of PCC was recorded and transcribed, and was made a thematic analysis of the content of the sessions. The analysis was performed by two reviewers independently. The final sample consisted of 9 patients. Result: The results indicate that in general there is a good adequacy of the constructs worked in therapy and the model. However, it will be necessary to include the issue of "interpersonal relationships", very important for the patient and not included in the model. In addition, information of the main concerns of patients, difficulty performing some tasks proposed and positive feedback of psychotherapy and its participation, were obtained. Conclusions: This pilot study provides relevant information to adjust and improve the initial proposed model of PCC.

Keywords: Psychotherapy; compassion; mindfulness; Cancer; psycho-oncology; emotional distress; qualitative research.

Sumario. 1. Introducción 2. Método 3. Análisis de los datos 3.1. Criterios de relevancia y adecuación 4. Resultados 5. Discusión 6. Referencias bibliográficas.

Cómo citar: Prieto Larroy C, Chamorro López J, Fernández Castro J, Gil Moncayo FL. Análisis cualitativo de temas construidos y emergentes a partir de un experiencia piloto de Psicoterapia Centrada en la Compasión (PCC) en pacientes con cáncer avanzado. Psicooncología 2017; 14: 149-158. 10.5209/ PSIC. 55818

\section{Introducción}

La esperanza de vida en la última década ha aumentado notablemente y con ella la prevalencia de enfermedades crónicas ${ }^{(1)}$. El $75 \%$ de la población fallece hoy con una o más enfermedades crónicas en progresión, con pronóstico de vida limitado e importante deterioro ${ }^{(2)}$. Una de las enfermedades crónicas más prevalentes y de gran impacto es el cáncer, principal causa de muerte a nivel mundial, y segunda causa de muerte en España ${ }^{(3)}$.

Pese al avance de la medicina en los tratamientos oncológicos y los programas de prevención, la mayoría de pacientes diagnosticados por cáncer acabará falleciendo por la enfermedad o como consecuencia de los efectos o complicaciones del tratamiento ${ }^{(4)}$.

Cuando la enfermedad alcanza el punto en que no es curable pero sí tratable, decimos que el paciente se encuentra en situación de enfermedad avanzada ${ }^{(2)}$. El impacto de la noticia de no curación, junto a el progreso de la enfermedad, enfrentan al paciente a una serie de cambios en las esferas física, psicológica, social y espiritual, que traen consigo gran malestar y alteraciones de la calidad de vida. En esta situación, es frecuente, que los pacientes experimenten sentimientos de pérdida de sentido, desesperanza, soledad, miedo, tristeza, ansiedad, rumiaciones y sintomatología depresiva, entre otros. Siendo uno de los objetivos principales el control emocional y la integración de la enfermedad y su pronóstico, que se aborda a través de la psicoterapia ${ }^{(5)}$.

A nivel de psicoterapia, son conocidos los beneficios del desarrollo de los constructos terapéuticos de "compasión" y "autocompasión" para mejorar el bienestar emocional. La práctica budista lleva promoviendo el desarrollo de estos constructos desde hace miles de años, junto a la atención consciente (mindfulness), para lograr un mejor bienestar personal. 
La palabra "compasión" proviene de la palabra latina compati que significa "sufrir con", aunque la definición más conocida es la del Dalai Lama que definió la compasión como "una sensibilidad hacia el sufrimiento del yo y de los otros junto con un compromiso profundo para tratar de aliviarlo $^{(6)}$.

Para este proceso de aceptar el sufrimiento en vez de evitarlo, es necesario desarrollar una serie de atributos, como son la simpatía, la tolerancia al malestar emocional, la empatía, el no juzgar, el autocuidado, la sensibilidad y habilidades de razonamiento y atención. Además, través de estas prácticas compasivas, podemos generar una sensación de seguridad que ayuda en el desarrollo de la conciencia plena (mindfulness) y la aceptación ${ }^{(7)}$.

Recientemente Paul Gilbert ${ }^{(8,9)}$ ha desarrollado un modelo de Terapia Centrada en la Compasión (Compassion Focused Therapy, CFT), dirigida a personas con problemas crónicos y complejos de salud mental ligados a la vergüenza y la autocrítica. El enfoque CFT, aunque toma elementos prestados de las enseñanzas budistas, tiene un enfoque evolutivo, neuro-científico y psicosocial, vinculado a la psicología del cuidado. Sentirse cuidado, seguro y compartir un sentido de pertenencia, no sólo está asociado a una baja tendencia a la depresión, ansiedad y estrés, sino que se relaciona con un perfil neuro-hormonal que se corresponde con niveles elevados de endorfinas y oxitocinas ${ }^{(10,11)}$. Gilbert ${ }^{(8,9)}$ propone que los psicoterapeutas se centren en buscar el modo de estimular la capacidad para experimentar emociones positivas asociadas con la tranquilidad, la seguridad y el bienestar en sus pacientes, a través de desarrollar la compasión hacia uno mismo y hacia los demás.

A partir del trabajo de Paul Gilbert ${ }^{(7-9)}$, y de los trabajos de otros autores cómo Choden $^{(7)}$, Kristin Neff ${ }^{(2,13)}$, Christoffer Germer ${ }^{(14)}$, Rob Nairn ${ }^{(15)}$, y William Breitbart ${ }^{(16,17)}$, desde el servicio de consultas externas del Instituto Catalán de Oncología de Duran i Reinals, se está desarrollando un modelo de Psicoterapia grupal Centrada en la Compasión (PCC), enfocada a pacientes con cáncer en fase avanzada. Con este modelo se pretende disminuir los niveles de malestar emocional (distress), y favorecer el crecimiento personal y espiritual de los pacientes en situación de enfermedad oncológica avanzada que acuden a las consultas externas de este dispositivo.

El Modelo de PCC que se propone tiene una orientación cognitivo-existencial y trabaja los constructos de "Compasión" y "Autocompasión" a través de técnicas cognitivas, conductuales y narrativas, así como la atención plena (mindfulness). Las primeras medidas de evaluación son compasión, autocompasión y malestar emocional; las segundas medidas de evaluación son aceptación, rumiación cognitiva, espiritualidad (sentido y propósito), crecimiento personal, atención plena (mindfulness) y seguridad.

Previo a la evaluación de la eficacia del presente modelo, se requiere evaluar cada uno de los componentes que forman la psicoterapia, con el fin de valorar su adecuación y relevancia en este tipo concreto de pacientes, así como el grado en que los constructos que trabaja PCC son de interés para los pacientes. Para ello, se lleva a cabo el presente estudio piloto de análisis cualitativo del contenido que surge a lo largo de un ciclo de 8 sesiones de PCC, y que tiene como objetivos específicos:

1. Valorar la adecuación de los temas propuestos en la terapia (si son de relevancia para el paciente)

2. Explorar nuevos temas no contemplados por la terapia, que puedan ser relevante para el paciente.

3. Valorar la consistencia global del modelo a nivel de estructura, tareas, temas, etc. 


\section{Método}

La muestra se formó a partir de los pacientes que acudían a consulta externa del Hospital Duran i Reynals, con el psicooncologo, quien valoraba y ofrecía su inclusión en el grupo.

Los criterios de inclusión fueron:

- Ser mayor de edad.

- Estar en un proceso de enfermedad avanzada (estadio IV).

- Estar en un estado físico y psicológico que permita seguir el ritmo de las sesiones.

- Que haya conciencia de enfermedad y gravedad.

- Que el psicooncólogo en la terapia individual previa haya valorado que por su sintomatología es apto para el grupo y se puede beneficiar de éste.

- Que el paciente aceptara participar voluntariamente.

Para el estudio piloto la terapia fue ofrecida a un total de 18 pacientes que acudieron a consultas externas del Hospital Duran i Reynals, en el periodo de tiempo que duró el estudio. De los pacientes a los que se les ofreció participar en el grupo aceptaron un total de 12, de los cuales sólo 9 acabaron el ciclo de 8 sesiones, uno por incompatibilidad de horarios, otro por empeoramiento de la enfermedad y necesidad de ingreso, y un tercero del cual se desconocen las causas.

El grupo tiene una estructura abierta, en el que un paciente puede incorporarse en cualquiera de las sesiones, por lo que el número de participantes fue variable, con un número medio de 6 pacientes por sesión, variando entre 4 y 8 . Una vez incorporado, se espera que el paciente complete el ciclo de 8 sesiones. Al finalizar el ciclo el paciente puede optar por seguir viniendo al grupo iniciando nuevamente el ciclo de 8 sesiones, estos pacientes pasan a llamarse "pacientes expertos". Se usa la figura del "paciente experto" para favorecer la acogida de nuevos participantes, ya que ayudan a acoger y favorecer una rápida normalización e integración de los nuevos pacientes.

\section{Características y estructura de las sesiones:}

El modelo consta de 8 sesiones estructuradas de 90 minutos, de frecuencia semanal. Con la siguiente estructura:

$1^{\circ}$. Asentar y aclarar la mente. Apertura: 5 minutos de meditación centrada en la respiración.

$2^{\circ}$. Presentación de nuevos miembros al grupo y ausencias.

$3^{\circ}$. Checklist de salud y tratamientos médicos.

$4^{\circ}$. Revisión de la sesión anterior. Tareas, dudas y comentarios.

$5^{\circ}$. Nueva sesión. Pregunta reflexiva sobre el constructo a trabajar en la sesión.

$6^{\circ}$. Practica a realizar durante la sesión (actividad, ejercicio, meditación, etc.).

$7^{\circ}$. Resumen de la sesión. Preguntas y comentarios.

$8^{\circ}$. Entrega de tareas y registros para casa.

$9^{\circ}$. Asentar y aclarar la mente. Cierre: 5 minutos de meditación centrada en la respiración.

A lo largo de las sesiones se trabajan constructos terapéuticos como "aceptación", "compasión", "autocompasión" o "seguridad", junto a la atención plena (mindfulness). 


\section{Análisis de los datos}

El análisis cualitativo de la información se realizó por medio de la técnica de análisis temático, mediante el programa ATLAS.TI (versión 7.14.5). Para ello se grabó en video un ciclo de 8 sesiones de grupo que forman la terapia, y posteriormente se trascribieron para realizar el análisis cualitativo de las temáticas que surgían.

Las trascripciones fueron analizadas independientemente por dos evaluadores. Se analizaron todos los temas que surgieron a lo largo de las sesiones y se agruparon por temática. Todo lo referente a un tema formó una categoría. Se dio a cada categoría un nombre que definiera todos los temas que englobaba y se especificaron los subtemas que incluyen esa categoría.

Luego se compararon los "temas" o categorías resultantes con los diferentes constructos que propone el guion de la terapia. Los temas que coincidan con temas propuestos por la terapia fueron considerados temas "construidos", mientras que los temas que surgieron y que no estaban propuestos por la terapia, fueron considerados temas "emergentes".

Posteriormente se pusieron en común los resultados que había obtenido cada evaluador, categorías de temas, subtemas, nombre que se le dio a cada categoría de temas, y temas "construidos" y "emergentes" (ver la tabla 1). Se compararon los resultados y en los casos donde había discrepancia se llegó a un acuerdo con la ayuda de un tercer evaluador experto en la materia (ver la tabla 2).

Tabla 1. Temas resultantes del análisis individual de los dos evaluadores.

\begin{tabular}{|l|l|}
\hline \multicolumn{1}{|c|}{ TEMAS EVALUADOR 1 } & \multicolumn{1}{c|}{ TEMAS EVALUADOR 2 } \\
\hline Diagnóstico & \multirow{2}{*}{ Tratamiento, pruebas, evolución enfermedad } \\
\hline Pronóstico enfermedad & \\
\hline Tratamiento / pruebas & Compasión \\
\cline { 2 - 2 } Compasión & Ser compasivo con los demás \\
\hline Autocompasión & Autocompasión \\
\hline Aceptación & Aceptación \\
\hline Base segura & Nuestra base segura \\
\hline Meditación & Meditación / atención plena \\
\hline Tareas / ejercicios & Tareas \\
\hline Síntomas físicos & Síntomas físicos \\
\hline Estado emocional & Síntomas emocionales \\
\hline Sentido & Legado \\
\hline Recursos & Afrontamiento sufrimiento/ miedos/ \\
\hline Miedos / preocupaciones & limitaciones \\
\hline Como la enfermedad nos ha cambiado & \\
\hline Meta-terapia & Meta-terapia \\
\hline Relación / comunicación con los demás & Relación con los otros \\
\hline
\end{tabular}


Tabla 2. Temas finales acordados a partir de los análisis individuales de los dos evaluadores.

\begin{tabular}{|c|c|}
\hline $\begin{array}{c}\text { TEMAS } \\
\text { CONSTRUIDOS }\end{array}$ & SUBTEMAS \\
\hline COMPASIÓN & $\begin{array}{l}\text { ¿Qué es ser compasivo? ¿Cómo expresamos la compasión? } \\
\text { ¿Qué nos impide ser compasivos con los demás? }\end{array}$ \\
\hline AUTOCOMPASIÓN & $\begin{array}{l}\text { ¿Qué es ser autocompasivos? ¿Qué nos impide ser } \\
\text { autocompasivos? ¿Cómo podemos promover nuestra } \\
\text { autocompasión? ¿Qué nos impide aceptar la compasión de los } \\
\text { demás? }\end{array}$ \\
\hline ACEPTACIÓN & $\begin{array}{l}\text { ¿Qué es aceptar? ¿Por qué nos cuesta aceptar? ¿Qué cosas } \\
\text { nos cuesta aceptar? }\end{array}$ \\
\hline $\begin{array}{l}\text { ATENCIÓN PLENA } \\
\quad \text { (mindfulness) }\end{array}$ & $\begin{array}{l}\text { Dificultades de la meditación, beneficios de la meditación, } \\
\text { estrategias que usan los pacientes para meditar, evolución en el } \\
\text { hábito de meditar }\end{array}$ \\
\hline $\begin{array}{l}\text { RUMIACIÓN } \\
\text { COGNITIVA }\end{array}$ & $\begin{array}{l}\text { Miedos y preocupaciones sobre las limitaciones, la muerte/ final } \\
\text { de la existencia, el sufrimiento propio y el de los seres queridos }\end{array}$ \\
\hline $\begin{array}{l}\text { SINTOMATOLOGÍA } \\
\text { FÍSICA }\end{array}$ & $\begin{array}{l}\text { Sintomas fisicos y efectos secundarios del tratamiento y las } \\
\text { intervenciones }\end{array}$ \\
\hline $\begin{array}{l}\text { MALESTAR } \\
\text { EMOCIONAL }\end{array}$ & $\begin{array}{l}\text { Sentimientos de tristeza, labilidad emocional, rabia, } \\
\text { irritabilidad, enfado, depresión, impotencia, desconcierto, etc. }\end{array}$ \\
\hline BASE SEGURA & $\begin{array}{l}\text { ¿Qué o quién nos da paz/ seguridad? ¿Dónde nos sentimos } \\
\text { seguros? }\end{array}$ \\
\hline SENTIDO & $\begin{array}{l}\text { Fuentes sentido pasadas, presentes y futuras; fuentes de } \\
\text { esperanza y pérfida de sentido y deseos de adelantar la muerte }\end{array}$ \\
\hline $\begin{array}{l}\text { TRANSFORMACIÓN } \\
\text { PERSONAL }\end{array}$ & $\begin{array}{l}\text { Redistribución de necesidades/prioridades, Cambios en los } \\
\text { valores/ creencias, Cambios personales (forma de ser) }\end{array}$ \\
\hline $\begin{array}{c}\text { ESTRATEGIAS/ } \\
\text { RECURSOS DE } \\
\text { AFRONTAMIENTO }\end{array}$ & $\begin{array}{l}\text { Estrategias, capacidades, habilidades, recursos que utilizas } \\
\text { para afrontar la enfermedad, las limitaciones, miedos, etc. }\end{array}$ \\
\hline META-TERÁPIA & $\begin{array}{l}\text { Beneficios asistencia grupo, Como nos ha cambiado la } \\
\text { participación en el grupo, Información sobre el grupo }\end{array}$ \\
\hline CHECKLIST & $\begin{array}{l}\text { Presentaciones, Ausencias, Éxitus, Diagnóstico, proceso } \\
\text { diagnóstico, Evolución de la enfermedad, Tratamientos, } \\
\text { pruebas, Creencias eficacia/no eficacia tratamiento }\end{array}$ \\
\hline TAREAS CASA & $\begin{array}{l}\text { Valoraciones positivas y beneficios de las tareas, y valoraciones } \\
\text { negativas o dificultades de las tareas }\end{array}$ \\
\hline $\begin{array}{c}\text { TEMAS } \\
\text { EMERGENTES }\end{array}$ & SUBTEMAS \\
\hline $\begin{array}{l}\text { RELACIÓN } \\
\text { INTERPERSONAL }\end{array}$ & $\begin{array}{l}\text { Lo que me molesta de los demás, Lo que espero o quiero de } \\
\text { los demás, Sentimientos de incomprensión, falta de apoyo y } \\
\text { soledad, Los demás no saben cómo ayudarnos }\end{array}$ \\
\hline
\end{tabular}

\subsection{Criterios de relevancia y adecuación}

- Se consideró los temas construidos o temas que propone la terapia eran relevantes para los pacientes si los pacientes los desarrollan una vez propuestos por el psicooncólogo. Y si no surgen muchos temas emergentes. 
- Consideraremos que el tema emergente es relevante si surge varias veces a lo largo de las sesiones y los pacientes expresan que es un tema que les preocupa.

- Consideraremos que la terapia tiene una buena consistencia si no surgen muchos temas emergentes (ya que significaría que hay otros temas que para los pacientes son más relevantes que los que propone la terapia), si los pacientes valoran positivamente la terapia, si se realizan las tareas, y si la mayoría de pacientes terminan el ciclo de las 8 sesiones y si los casos de abandono no se deben a la propia terapia.

\section{Resultados}

El análisis cualitativo del contenido de las 8 sesiones de PCC evaluadas, dio como resultado un conjunto de temas considerados relevantes para los pacientes que surgieron a lo largo las 8 sesiones.

En el primer análisis individual llevado a cabo por los dos evaluadores de forma independiente (tabla 1), la clasificación de los temas fue bastante similar entre evaluadores, aunque hubo pequeñas diferencias en cómo se han denominado a las distintas categorías o temáticas, y en la división. Tras la puesta en común de los resultados y la revisión del tercer evaluador experto en la materia, se obtuvo una clasificación definitiva de 15 categorías o "temas" (tabla 2), 14 de ellos fueron considerados construidos (ya que coincidían con los planteados desde el modelo) y 1 único tema fue considerado emergente, el de "las relaciones interpersonales", no contemplado el modelo.

\section{Discusión}

Uno de los principales objetivos del presente estudio piloto era ver el grado de adecuación de los constructos propuestos, así como explorar si surgían nuevos temas de relevancia no contemplados en el modelo. A partir de los criterios de adecuación establecidos, podemos considerar que los constructos que trabaja la terapia son de relevancia para el paciente ya que los pacientes los desarrollan y se identifican con ellos.

Además, ha surgido un nuevo tema que no estaba contemplado en el modelo, el de "relaciones interpersonales", que ha resultado ser de gran importancia para los pacientes. En este tema se engloba todo lo relacionado con la relación, comunicación y las expectativas que tienen los pacientes con sus seres más allegados, por lo general la familia.

Entre las cosas que los pacientes expresan que más les molestan de los demás encontramos, por ejemplo, que les den consejos en vez de escucharlos: “¿Cuándo nos escuchó la familia?, Nunca... la familia nos aconseja... la familia nos dice haz esto, haz lo otro, pro que no hiciste aquello, pero no nos escucha..." (P1). Y que le resten importancia a lo que les pasa: "me molesta que le quiten importancia a lo que tienes... a mi me molesta mucho eso, cuando a lo mejor un día tengo mucho dolo y me cuesta moverme y te dicen: jvenga va que no será para tanto, que eso no es nada!, ¿Cómo que no es nada? ¿Quién dice que no es nada?, me molesta mucho...” (P2).

Algunos pacientes también dicen sentirse incomprendidos por las personas relevantes de su entorno: "tengo la sensación de que mi marido no me escucha y esas cosas... o sea que nunca me he sentido comprendida siquiera... [...] y yo lo noto a faltar... el poder abrirte y que la otra persona te comprenda..." (P4). 
También cabe destacar, que los pacientes son conscientes de que muchas veces las personas de su entorno no saben cómo ayudarles: "es que a veces cuanto más quieren ayudarte más te agobian" (P2); "yo creo que a veces ellos tampoco saben cómo tranquilizarte, como comunicarse, utilizan frases hechas, que ni lo piensan... pero te sientan mal" (P6).

Los pacientes también expresaron no compartir y expresar sus emociones, preocupaciones, lo que les molesta o necesitan con los familiares, principalmente para evitarles más sufrimiento o porque no saben cómo hacerlo. Todo esto genera gran malestar tanto en los pacientes como en sus familiares, por lo que consideramos de gran relevancia incorporar este tema de cara a futuras intervenciones.

Pese a que el grupo facilita la ventilación y expresión emocional, es importante trabajar a través de la terapia los temas de comunicación, relación e intercambio de emociones con los seres queridos como estrategia de intervención terapéutica, ya que la familia es la principal fuente de apoyo del paciente. Mejorar estos niveles de estrés que se generan a la hora de relacionarse ayudará al afrontamiento conjunto de la enfermedad y a disminuir los posibles sentimientos de culpa de paciente y familia. La comunicación por parte del paciente de cómo se siente y de lo que necesita, favorece que los familiares puedan ofrecer una mejor atención, sintiéndose a su vez más eficaces y reconfortados ${ }^{(18)}$.

Por otra parte, este estudio también ha permitido observar algunos aspectos relevantes a tener en cuenta de cara a mejorar el modelo. En primer lugar se ha visto que es necesario definir bien los constructos con los que se trabaja en la terapia, ya que los pacientes pueden tener una definición o asociación diferente que dificulte el trabajo en ellos y su asimilación ${ }^{(19)(20)}$. Esto es frecuente con el constructor de "autocompasión" que algunos pacientes asocian con autocompadecerse, o con el constructo "aceptación" que puede ser entendido por los pacientes como resignación o rendirse.

En segundo lugar, se ha observado que algunos pacientes tienen dificultades a la hora de hacer algunas de las actividades propuestas, como son la carta reflexiva o el proyecto de legado. La explicación que los pacientes dan es, que les supone mucha carga emocional y se vienen abajo, en el primer caso, y que lo ven como una despedida en el segundo caso.

Entre las principales preocupaciones que han expresado los pacientes que más malestar les generan encontramos el dolor, las limitaciones, el miedo al sufrimiento ante la muerte, tanto propio como de los seres queridos, y la relación actual con los seres más allegados. Estas preocupaciones coinciden con las encontradas por otros autores en esta fase del proceso ${ }^{(21-23)}$.

También expresan que el grupo les ha permitido compartir las preocupaciones y sentimientos que no comparten con sus seres queridos, además, al ser un grupo formado por personas que están pasando por la misma situación se han sentido comprendidos. Esto refuerza nuestra decisión de una modalidad de terapia grupal, ya que el mismo grupo es terapéutico ya que permite la ventilación emocional y genera sentimientos de comprensión, pertenencia, que mejora la autoestima ${ }^{(24)}$.

Todos los pacientes han valorado positivamente la terapia y su participación en el grupo, e indican que al finalizar el ciclo de las 8 sesiones han disminuido las rumiaciones cognitivas, el malestar emocional y el estrés, sin embargo, estos datos no son objetivos por lo que será necesario pasar instrumentos que evalúen estas variables para verificar si realmente se dan cambios en estas medidas. 
Entre las limitaciones de este estudio identificamos que, al partir de una terapia estructurada para valorar la relevancia de los temas propuestos y la existencia de temas emergentes no contemplados en la terapia, hace que en parte los temas que surgen a lo largo de la terapia ya hayan sido condicionados, esto puede dejar menos margen para que surjan otros temas.

Otra limitación que encontramos es que el grado de participación de cada paciente también fue variable, ya que los pacientes son libres de intervenir. Encontramos, por lo tanto, que las opiniones y reflexiones extraídas sobre cada tema no pertenecen a la totalidad de los pacientes por igual.

Como última limitación decir que el presente estudio no evalúa efectividad, por lo que no podemos concluir aun que el modelo sea eficaz.

A nivel de implicaciones, el presente análisis cualitativo ha permitido valorar el grado de adecuación del contenido de la terapia propuesta a las necesidades reales de los pacientes, así como detectar dificultades y posibles mejoras que complementen el presente modelo de PCC.

A nivel del modelo, es una psicoterapia diseñada específicamente para pacientes con cáncer en fase avanzada a partir de sus preocupaciones y necesidades, por lo que se espera que englobe y aborde la mayor parte de la sintomatología psicoemocional que presentan estos pacientes, y se ajuste lo máximo posible a sus necesidades.

También propone un enfoque algo diferente a otras terapias, desde la perspectiva de aceptar y permitir los sentimientos en cada momento, frente a otros enfoques de afrontamiento más activo. Este enfoque a su vez, promueve la compasión, la aceptación del sufrimiento y la disminución de la autocrítica, para lograr la calma interior y el estar bien con uno mismo.

Como conclusión decir que hay una buena adecuación de los constructos y el contenido de la terapia. Que será necesario incorporar el tema de las "relaciones interpersonales" al presente modelo de cara a futuras intervenciones. Así como explorar más a fondo las dificultades y elementos que interfieren en algunas tareas que se proponen, y ver cómo abordarlas para que los pacientes se puedan beneficiar de éstas. Por último, queda pendiente evaluar la eficacia del modelo con instrumentos de medida válidos, y su eficacia frente a otros modelos.

\section{Referencias bibliográficas}

1. Instituto Nacional de Estadística. Sostenible D. Salud (actualizado 20 mayo 2015) 4.1 Esperanza de vida. (actualizado 20 mayo 2015). 2015; 1-13. [Acceso 1 enero 2017]. Disponible en: http://www.ine.es/ss/Satellite?L=es_ES\&c=INESeccion_C\&cid=12599263 80048\&p=1254735110672\&pagename=ProductosYServicios/PYSLayout

2. Gómez-Batiste X, González-Olmedo MP, Maté J, González Barboteo J, Duran A, Codorniu $\mathrm{N}$, et al. Principios básicos de la atención paliativa de personas con enfermedades crónicas evolutivas y pronóstico de vida limitado y sus familias. "Observatorio "Qualy"- Centro Colaborador de la OMS para Programas Públicos de Cuidados Paliativos del Institut Català d' Oncologia, 2011.

3. Instituto Nacional de Estadística. Defunciones según la causa de muerte. Año 2013. INE [internet], 2015;1-8. [Acceso 1 de enero de 2017]. Disponible en: http://www.ine.es/ prensa/np896.pdf 
4. Ahmedzai SH, Costa A, Blengini C, Boscch A, Sanz-Ortiz J, Ventafridda V, et al. International working group convened by the European School of Oncology. A new international framework for Palliative Care. Eur J Cancer 2004; 40:192-200. Doi. 10.1016/j.ejca.2004.06.009

5. Gil F, Sirgo A, Lluch P, Maté J, Estradé E. Intervención psicológica en pacientes de cáncer. En Gil, editor. Counselling y Psicoterapia en Cáncer. España: Elsevier, 2014.p.401-24.

6. The Dalai Lama. The power of compassion: A Collection of Lectures by His Holiness the XIV Dalai Lama.Thorsons, Kindle Edition, 2013.

7. Gilbert P, Choden. Mindful Compassion: How the science of compassion can help you understand your emotions, live in the present, and connect deeply with others. Oakland, CA: New Harbinger Publications, 2014.

8. Gilbert P. An introduction to compassion focused therapy in cognitive behavior therapy. Inter J Cognitive Ther 2010;3: 97-112. Doi: 10.1521/ijct.2010.3.2.97

9. Gilbert P. Terapia centrada en la compasión: características distintivas. Bilbao: Desclée de Brouwer, 2015.

10. Carter CS. Neuroendocrine perspectives on social attachment and love. Psychoneuroendocrinology 1998; 23: 779-818. Doi:10.1016/S0306-4530(98)00055-9

11. Panksepp J. Affective neuroscience: The foundations of human and animal emotions. Oxford University Press, 1998.

12. Neff K. Self-compassion: An alternative conceptualization of a healthy attitude toward oneself. Self and identity 2003; 2: 85-101. 10.1080/15298860309032

13. Neff K. Self-Compassion: stop beating yourself up and leave insecurity behind. New York: Harper Collins, 2011.

14. Germer CK. The mindful path to self-compassion: Freeing yourself from destructive thoughts and emotions. New York: Guilford Press, 2009.

15. Nairn R. Diamond mind: A psychology of meditation. Boston: Shambhala Publications, 2015.

16. Breitbart W, Rosenfeld B, Gibson C, Pessin H, Poppito S, Nelson C, et al. Meaning-centered group psychotherapy for patients with advanced cancer: a pilot randomized controlled trial. Psychooncology 2010;19:21-8. Doi: 10.1002/pon.1556.

17. Breitbart W, Rosenfeld B, Pessin H, Applebaum A, Kulikowski J, Lichtenthal WG. Meaningcentered group psychotherapy: An effective intervention for improving psychological well-being in patients with advanced cancer. J Clin Oncol 2015:33: 749-54. Doi: 10.1200/ JCO.2014.57.2198

18. Baider L. Cáncer y familia: aspectos teóricos y terapéuticos. Inter J Clin Health Psychol 2003;3:505-20.

19. Miró MT. La atención plena (mindfulness) como intervención clínica para aliviar el sufrimiento y mejorar la convivencia. Rev Psicoterap 2006;17: 31-76.

20. Pérez MA, Botella L. Conciencia plena (mindfulness) y psicoterapia: concepto, evaluación y aplicaciones clínicas. Rev Psicoterap 2007;17: 77-120.

21. Fernández C, Padierna C, Amigo I, Pérez M, Gracia J M, Fernández R et al. Calidad de vida informada por pacientes oncológicos paliativos: Relaciones con el informe médico y estado emocional. Index Enferm [Internet]. 2006 [Acceso 24 de marzo de 2017]; 15:30-4. Disponible en: http://scielo.isciii.es/scielo.php?script=sci_arttext\&pid=S1132-12962006000100007\&lng=es

22. Krikorian A. Valoración del sufrimiento en pacientes con cáncer avanzado. Psicooncología 2008;5:257-64.

23. Krikorian A, Limonero JT. Factores asociados a la experiencia de sufrimiento en pacientes con cáncer avanzado. Av Psicol Latinoam 2015;33:423-38. Doi: 10.12804/revistas.urosario. edu.co/apl/a.3247

24. Bárez M, Blasco T, Fernández Castro J. La inducción de sensación de control como elemento fundamental de la eficacia de las terapias psicológicas en pacientes de cáncer. An Psicol 2003;9:235-46. 